\title{
TENSILE DEFORMATION INDUCED TEXTURE TRANSFORMATION IN AUSTENITIC STAINLESS STEEL
}

\author{
S. GRIGULL \\ Department of Earth and Planetary Science, University of California, Berkeley, CA 94720; \\ E-mail: grigull@37.com
}

(Received 30 June 2003)

\begin{abstract}
Texture patterns of the starting $\gamma$ and transformed $\alpha$ structural phases were obtained from AISI 304 stainless steel sheets subjected to varying levels of tensile deformation using high energy X-ray diffraction in combination with the texture enhanced Rietveld method. The use of this method allows the simultaneous determination of the orientation distribution functions (ODF) of both phases, even for small $\alpha$-martensite fractions of the order of $5 \%$. The texture patterns are analyzed in terms of the crystallographic orientation relation between the starting and transformed phases and the preferential formation of certain variants of this relation.
\end{abstract}

Keywords: Phase transformation; Rietveld method; Texture patterns; Tensile deformation

\section{INTRODUCTION}

Due to its enormous practical importance the deformation induced $\gamma-\alpha$ transformation in ferrous alloys has been studied for more than 50 years, and numerous experimental and theoretical studies have been reported focusing on the transformation mechanism (Patel and Cohen, 1953; Angel, 1954; Bogers and Burgers, 1964, Olson and Cohen, 1975; Furubayashi, 1985). Renewed interest in a better understanding of this mechanism and its effect on texture evolution results from the need of reliable input parameters for the development of computer codes simulating various metallurgical forming processes (Ding et al., 2001). Since the $\gamma-\alpha$ transformation is martensitic the crystallographic orientation relation between the starting and transformed phases is necessarily a key element in any model of the transformation mechanism. Various studies on single crystal alloys subjected to both rolling and tensile loading conditions (Abe and Ito, 1967; Borik and Richman, 1967; Higo et al. 1974; Stone and Thomas, 1974; Kato and Mori, 1977; Sato et al., 1980; Miyaji and Furubayashi, 1990) are confirming that this orientation relation follows the Bain/Nishiyama-Wassermann/KurdjumovSachs models (Dahmen 1982) which yield very similar relative orientations that cannot be easily distinguished under typical experimental conditions. Comparatively few experimental work has been dealing with the problem of how the deformation induced $\gamma-\alpha$ transformation affects the crystallographic texture of polycrystals (tension: 
Jung, 1983; rolling: Chapellier et al., 1990; Raabe, 1997), although this question is of key importance for the quantitative determination of the martensite content by diffraction methods (Bonarski et al., 1991), and although texture measurements can provide invaluable statistical information on the still not too well understood phenomenon of variant selection. Variant selection is said to occur if not all of the crystallite orientations allowed by a particular orientation relationship plus parent crystal symmetry are observed in the transformation product with equal probability. For example, the Kurdjumov-Sachs (KS) orientation relation

$$
(111)_{\gamma}\left\|(011)_{\alpha} \quad[-110]_{\gamma}\right\|[1-11]_{\alpha}
$$

often associated with the $\gamma-\alpha$ transformation in ferrous alloys with low stacking fault energy such as stainless steel allows 24 symmetry equivalent orientation variants in the transformation product to be formed from each parent crystallite orientation. However, these 24 variants are never observed to occur all together in the martensite phase. Two types of criteria have been used with some success to explain at least in part experimental observations of variant selection in single crystal samples. The first is based on the Bain model in which the fcc-bcc lattice transformation is driven by a compression along one of the three $\langle 100\rangle$ crystallographic axes. The criterion states that those martensite orientation variants will be formed preferentially that are derived from austenite grains which experience a maximum compressive strain along one of their cube axes (Furubayashi, 1985; Olsen and Jesser, 1971). The second criterion is based on the shear transformation model proposed by Bogers and Burgers (1964). This criterion assumes that those variants form for which shear in the two $\{111\}\langle 211\rangle$ twin systems of the fcc lattice involved in the transformation is aided to a maximum degree by the deformation strain (Higo et al., 1974). In contrast, a phenomenological model assuming that martensite forms preferentially if the external strain aids in the shape deformation associated with the transformation has been less successful as a criterion for variant selection (Kato and Mori, 1977).

The availability of high energy synchrotron radiation for bulk sensitive diffraction measurements, along with the development of fast area detectors, has greatly enhanced the possibilities of quantitative texture analysis (Heidelbach et al., 1999; Wcislak et al., 2002). In parallel, the implementation of advanced texture models in Rietveld refinement codes has matured to a degree that meanwhile allows to extract complete texture information on multiphase systems with partly overlapping diffraction peaks from one or at most a few diffraction images (Lutterotti et al., 1997; Von Dreele, 1997), although this has not yet been demonstrated on synchrotron diffraction data. In the present work, the combination of these techniques is used to fill part of the gap in existing texture data on the $\gamma-\alpha$ transformation in uniaxially stretched austenite, with the focus on the effect of orientation variant selection on the martensite texture pattern.

\section{EXPERIMENT AND DATA PROCESSING}

Commercial grade AISI 304 rolled and annealed stainless steel sheets of $0.95 \mathrm{~mm}$ thickness were cut into a standard dog bone shape and exposed to different levels of uniaxial tension along the rolling direction (RD), using an INSTRON testing machine. The tests 
were performed at a low strain rate of $10^{-3} \mathrm{~s}^{-1}$. The texture measurements were carried out on the 11-ID B wiggler beamline at the Advanced Photon Source synchrotron ring at Argonne National Laboratory at a beam energy of $85 \mathrm{keV}$ and diameter of $0.2 \mathrm{~mm}$, respectively. Diffraction images were recorded in transmission geometry with the RD perpendicular to the primary beam, using a MAR 345 online image plate system. The counting time was $120 \mathrm{~s}$ per image. To improve the pole figure coverage images were taken at different sample tilt angles between $\pm 30^{\circ}$, with the RD as tilt axis. Figure 1 is a schematic of the $(111)_{\gamma}$ pole figure coverage obtained from a set of 7 diffraction images, with the tilt angle varying in $10^{\circ}$ steps. The incomplete pole figure coverage was compensated for by the use of several $h k l$ diffraction peaks in the range of $d^{*}=0.5-1.8 \AA^{-1}$, corresponding to $2 \theta$ angles between $4^{\circ}$ and $15^{\circ}$, in the texture evaluation. The orientation distribution function (ODF) was determined for each sample using a pole figure inversion algorithm based on the WIMV method (Matthies and Vinel, 1982) combined with Rietveld refinement, as implemented in the Rietveld code MAUD (Lutterotti et al., 1997). From the ODF, complete pole figures were recalculated for different $h k l$.

Prior to Rietveld refinement, the diffraction images were transformed from polar into cartesian coordinates, corrected for detector tilt, polarisation and absorption and converted from binary into ascii format. The conversion of the diffraction ring radii into $2 \theta$ angles was obtained from fitting the peak positions of a powder standard measured under identical conditions. Each image was divided into $362 \theta$ scans, corresponding to an azimuthal resolution of $10^{\circ}$. A total number of 252 scans, each corresponding to a conventional powder pattern, was used in the refinement procedure. Although the use of a single diffraction image (=36 scans) already gave satisfactory texture patterns

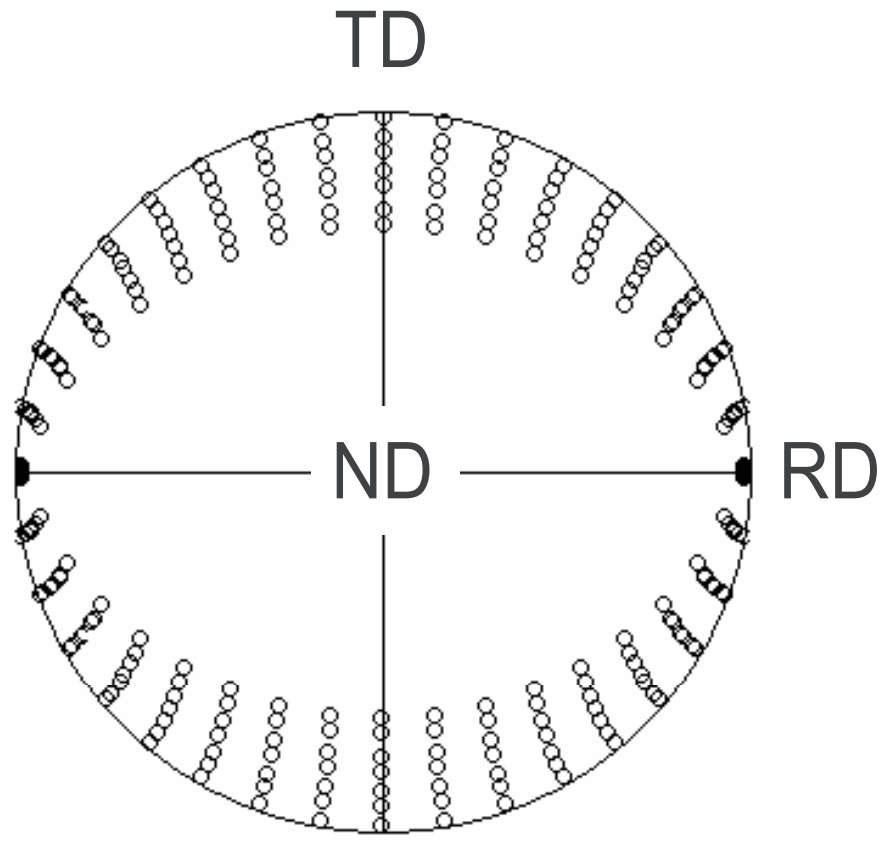

FIGURE 1 Pole figure coverage for the 111 austenite reflection in a measurement setup with 7 different tilt angles between $\pm 30^{\circ}$ and an azimuthal resolution of $10^{\circ}$. Tilt axis is parallel to the RD. 
for the dominating austenite phase multiple images taken at different tilt angles were necessary to obtain quantitative texture information for the martensite, due to both relatively low concentrations of about $1-20 \%$ and the lower number of martensite diffraction peaks within the measured $2 \theta$ range. Lattice parameters, lattice strains, phase fractions and microstructure parameters were refined simultaneously with the texture evaluation, to a final total weighted residual $R_{w p}$ on the order of $12-15 \%$. The main advantage of the Rietveld method over conventional pole figure analysis using integrated diffraction peak intensities is that in general information from more $h k l$ peaks is included in the analysis and that overlapping peaks from multiple structural phases can be handled without problems. Practical experience of the authors also shows that in the frequent case of limited experimental pole figure coverage (see Fig. 1) the Rietveld method results in more reliable texture patterns than the conventional method which can be explained by the fact that the texture weights are extracted from a physically constrained sample model rather than directly from the measured data.

\section{RESULTS AND DISCUSSION}

Recalculated pole figures obtained from the Rietveld refinement procedure for the $\gamma$ and $\alpha$ phases, respectively, are shown in Fig. 2. The texture patterns correspond to a true tensile strain of $\varepsilon=0.3$ and a resulting martensite content of about $5 \%$, the latter refined simultaneously with the texture evaluation. Tensile axis and RD are horizontal, and the normal direction (ND) is pointing out of the plane of the paper. In terms of ideal orientations, the austenite texture pattern (a) is dominated by a strong Copper-type (C) component $\{211\}\langle 111\rangle$ and a weaker Goss-type (G) component $\{011\}\langle 100\rangle$. Also present is a very weak Brass (B) component $\{011\}\langle 211\rangle$. The corresponding volume fractions of the three components as obtained from the

(a)

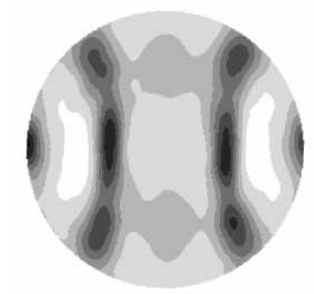

100

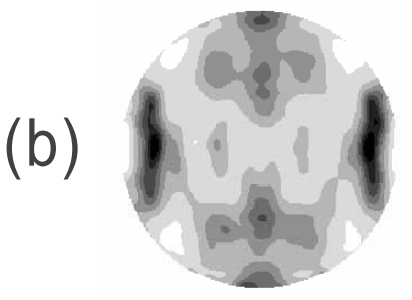

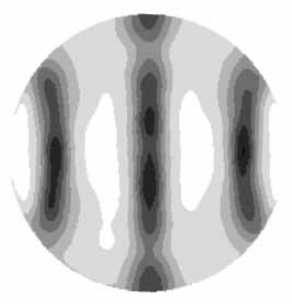

110

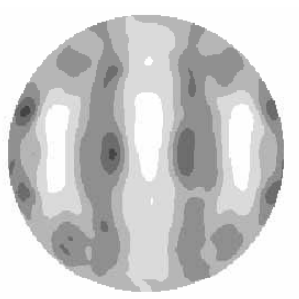

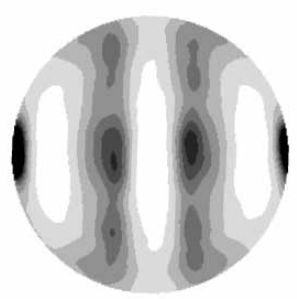

111

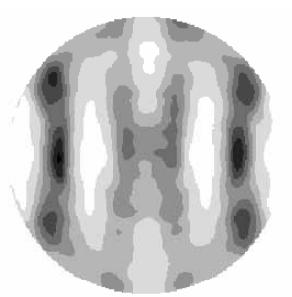

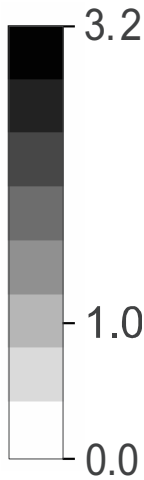

FIGURE 2 Complete pole figures recalculated from the ODF obtained from the diffraction data: (a) $\gamma$ phase (fcc) and (b) $\alpha$ phase (bcc). Equal area projection - linear intensity scale matched to $\alpha$. 
ODF were estimated to $\mathrm{C}: \mathrm{G}: \mathrm{B}=0.7: 0.23: 0.07$ where other orientations have not been accounted for. The texture pattern of the deformed sample is qualitatively similar to the initial rolling texture (not shown) but has a much stronger 111 pole maximum along the RD, and is closer to a fiber symmetry. Figure 3(a) is the inverse pole figure of the RD corresponding to the austenite pole figures in Fig. 2(a). The dominating 111 and 100 pole maxima are typical for fcc deformation textures developed under uniaxial tension and are believed to represent orientations stable with respect to deformation by slip or twinning (Bronkhorst et al., 1992). The texture patterns of both phases do not change qualitatively as a function of the applied strain in the range between $\varepsilon=0.2-0.5$ (not shown). Figure $3(\mathrm{~b})$ is the inverse pole figure of the RD for the martensite. The figure shows that in the transformed phase most grains are aligned with a $\langle 012\rangle$ crystal axis close to the RD. Minor fractions also occur with the RD along crystal directions between $\langle 012\rangle$ and $\langle 011\rangle$ and between $\langle 012\rangle$ and $\langle 113\rangle$. A more detailed analysis presented below indicates that the three corresponding pole maxima labeled $\mathrm{C}^{\prime}, \mathrm{G}^{\prime}$ and $\mathrm{B}^{\prime}$ in Fig. 3(b) can be associated with transformation products of the $\mathrm{G}, \mathrm{C}$ and $\mathrm{B}$ components, respectively.

For the computer analysis of the relative crystallographic orientation of the fcc and bcc phases, the starting texture (Fig. 2a) was approximated as a mixture of the 3 ideal $\mathrm{C}, \mathrm{G}$ and $\mathrm{B}$ components. Each component was described by an orientation matrix $g$ and all 24 possible bcc daughter orientations allowed by the KS model were calculated using suitable matrix operations. The ensemble of resulting 100, 110 and 111 crystal projections was compared to the experimental pole figures in Fig. 2(b), and orientation variants resulting in pole maxima not compatible with the experimental data were ruled out. Some of the variants showed compatibility but were not strictly necessary to reproduce qualitatively the $\alpha$ texture pattern in Fig. 2(b). These variants will be designated as "allowed" as opposed to variants required to explain the texture transformation which shall be referred to as "observed" variants.

In the case of the dominating $\mathrm{C}$ component, 8 out of the 24 possible $\mathrm{KS}$ variants proved to be either observed or allowed. Table I lists these variants with an identifying index used in the computer analysis, along with the relative orientations of the coinciding crystallographic planes and directions within the planes, respectively, and an approximate assignment of resulting martensite ideal orientations. The 8 variants are

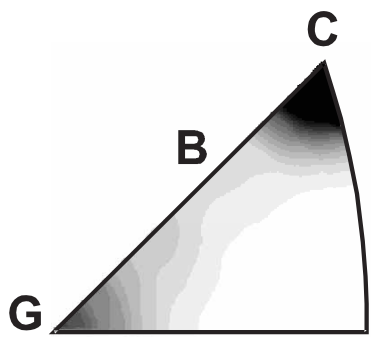

(a)
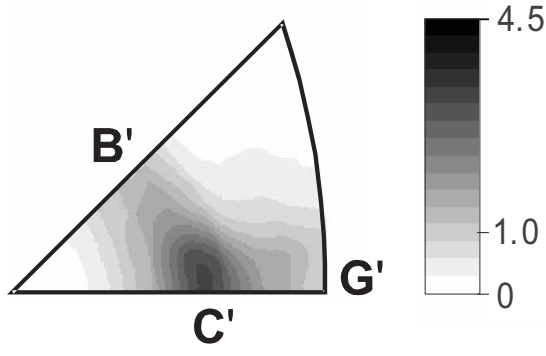

(b)

FIGURE 3 Inverse pole figures corresponding to the pole figures in Fig. 2, showing the probability distribution for different crystallographic directions to match the RD: (a) $\gamma$ phase and (b) $\alpha$ phase. 
TABLE I KS orientation variants allowed by the experimental $\alpha, \gamma$ texture patterns in Fig. 2. Variants marked with an asterisk are not required to reproduce the $\alpha$ texture pattern

\begin{tabular}{|c|c|c|c|}
\hline \multicolumn{4}{|c|}{$K S$ relation } \\
\hline $\begin{array}{l}\text { Variant } \\
\text { index }\end{array}$ & $\begin{array}{l}\text { Plane } \\
(\gamma) \|(\alpha)\end{array}$ & $\begin{array}{c}\text { Direction } \\
{[\gamma] \|[\alpha]}\end{array}$ & $\begin{array}{l}\text { Ideal }(\alpha) \\
\text { orientation }\end{array}$ \\
\hline $\begin{array}{l}3 \\
4\end{array}$ & $\begin{array}{l}(111)(011) \\
(111)(011)\end{array}$ & $\begin{array}{l}{[-110][-1-11]} \\
{[-101][-11-1]}\end{array}$ & $\begin{array}{l}\{012\}\langle 124\rangle \\
\{012\}\langle 124\rangle\end{array}$ \\
\hline $\begin{array}{l}14 \\
15^{*} \\
17^{*} \\
18\end{array}$ & $\begin{array}{l}(1-11)(011) \\
(1-11)(011) \\
(1-11)(011) \\
(1-11)(011)\end{array}$ & $\begin{array}{r}{[011][-1-11]} \\
{[-101][-1-11]} \\
{[011][-11-1]} \\
{[-101][-11-1]}\end{array}$ & $\begin{array}{l}\{012\}\langle 012\rangle \\
\{012\}\langle 123\rangle \\
\{012\}\langle 123\rangle \\
\{012\}\langle 012\rangle\end{array}$ \\
\hline $\begin{array}{l}21 \\
22\end{array}$ & $\begin{array}{l}(11-1)(011) \\
(11-1)(011)\end{array}$ & $\begin{array}{r}{[-110][-1-11]} \\
{[011][-11-1]}\end{array}$ & $\begin{array}{l}\{012\}\langle 124\rangle \\
\{012\}\langle 124\rangle\end{array}$ \\
\hline
\end{tabular}

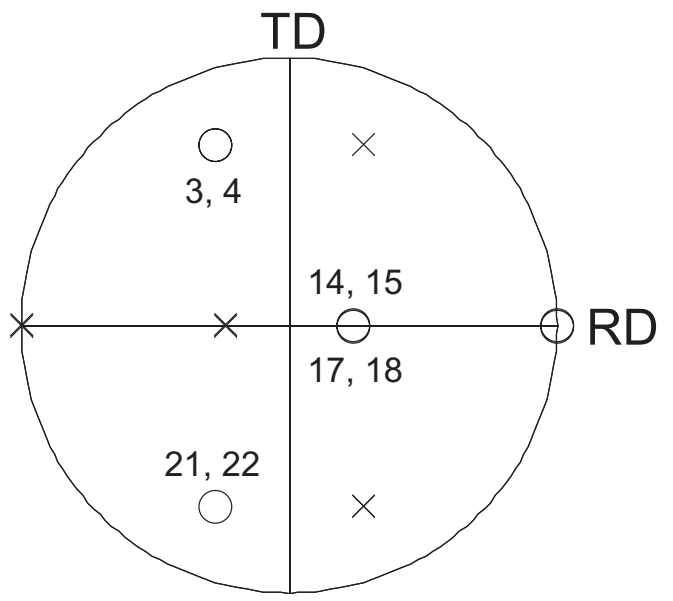

FIGURE 4 Location of different $\{111\}$ poles of the $\mathrm{C}$ texture component of the $\gamma$ phase relative to the RD, TD and ND. Numbers represent $\alpha$ grain orientations with a $\{011\}$ plane parallel to the respective $\{111\}_{\gamma}$ planes (see Table I).

grouped according to which of the 4 possible $\{111\}_{\gamma}$ planes are aligned to a $\{011\}_{\alpha}$ plane (dashed lines separate the three groups). Figure 4 shows the orientation of the 4 possible $\{111\}_{\gamma}$ poles corresponding to one of the two crystallite orientations forming the native $\mathrm{C}$ component (circles), the poles of the second orientation needed to preserve the sample symmetry are indicated by crosses. Numbers close to the $\{111\}_{\gamma}$ poles indicate which of the orientation variants in Table I has a $\{011\}_{\alpha}$ lattice plane normal aligned to the respective $\{111\}_{\gamma}$ poles. Notably, none of the observed or allowed variants has a $\{011\}_{\alpha}$ plane normal aligned parallel to the RD, corresponding to the $[-111]_{\gamma}$ direction. This is a strong indication that shear in the $\{111\}_{\gamma}$ planes plays an important role in the deformation induced martensite formation since the tensile strain along the RD does not induce any shear in the $(-111)_{\gamma}$ plane (see shear criterion in the introduction).

The 3 remaining $(111)_{\gamma},(1-11)_{\gamma}$ and $(11-1)_{\gamma}$ poles are each located at an angle of $70.6^{\circ}$ relative to the RD and are symmetrically equivalent with respect to the tensile axis. Accounting only for the 6 observed orientation variants, the variant selection 
for the $\mathrm{C}$ component appears to be determined by the uniaxial symmetry of the applied strain since two variants can be assigned to either of the 3 poles. However, this is no longer true if one allows for the possibility that the two allowed variants (no. 15, 17 in Table I) also occur in the transformation. This would relate 4 variants to the $(1-11)_{\gamma}$ pole as opposed to two variants assigned to the $(111)^{\gamma}$ and $(11-1)_{\gamma}$ poles, respectively. This difference could not be accounted for by the shear criterion and would have to be related to deformation history of the sample prior to application of the tensile strain, i.e. to the microstructure or residual elastic strains resulting from the initial rolling process. Note that a hypothetical variant selection including all 8 variants listed in Table I is in compliance with the sheet sample symmetry imposed by the rolling process: The $(111)_{\gamma}$ and $(11-1)_{\gamma}$ poles are symmetrically equivalent but not equivalent to the other two poles which in turn are not equivalent to each other.

In the case of the $\mathrm{G}$ component, 4 out of the $24 \mathrm{KS}$ variants were found to be observed. This is somewhat in contradiction with earlier work suggesting that the $G$ component may be stable against a deformation induced transformation into martensite (Goodchild et al., 1970; Raabe, 1997). The 4 variants result in ideal orientations close to $\{110\}\langle 111\rangle_{\alpha}$. The variant selection can be explained in part by the compression criterion (see Introduction). In the case of the G component two out of the $3\langle 100\rangle_{\gamma}$ axes actually experience an equal amount of compression during tensile loading - the two axes perpendicular to the tensile direction. According to the Bain model on which the compression criterion is based, the martensite grains originating from the transformation have one of their cube axes aligned parallel to the compression axis. The 4 observed KS variants have each a cube axis close to one of the two $\langle 100\rangle_{\gamma}$ axes of the $\mathrm{G}$ component which experience compression, and an equal number of variants is related to either of the two axes as required by the sample symmetry. No variant was found with a $\langle 100\rangle_{\alpha}$ axis close to the third $\langle 100\rangle_{\gamma}$ axis parallel to the tensile direction (RD), quite in accordance with picture that the tensile strain along this direction would rather hinder than aid in a uniaxial lattice compression.

The B component in the observed austenite texture pattern (Fig. 2a) is too weak to allow detailed conclusions on variant selection related to this particular texture component. Only 4 variants produced rather sharp maxima in locations with very low experimental pole densities and were ruled out. However, certain details in martensite pole figures obtained from the transformed sample (Fig. 2b) indicate that a third texture component apart from the $\mathrm{C}$ and $\mathrm{G}$ components discussed above must partially transform into martensite if the transformation is assumed to follow the KS orientation relation (1). Closer inspection of the inverse RD and ND inverse pole figures of the martensite phase (for RD see Fig. 3(b), ND not shown) suggests the presence of minor $\{332\}\langle 113\rangle_{\alpha},\{111\}\langle 112\rangle_{\alpha}$ and $\{112\}\langle 113\rangle_{\alpha}$ components in the martensite texture, and these orientations can indeed be derived from the $\mathrm{B}$ component of the austenite texture using relationship (1). The first two of these 3 components have been reported to occur in austenitic stainless steel after cold rolling (Raabe, 1997).

Figure 5 shows simulated $(100)_{\alpha},(110)_{\alpha}$ and $(111)_{\alpha}$ pole figures obtained from the superposition of the pole maxima obtained from the 3 ideal $\mathrm{C}, \mathrm{G}$ and $\mathrm{B}$ austenite texture components, using $8 \mathrm{KS}$ orientation variants for the $\mathrm{C}$ component (see Table I) as well as 4 variants for the $\mathrm{G}$ component and 20 variants for the weak B component (see discussion above). Each variant was weighted with the estimated volume fraction of the parent $\mathrm{C}, \mathrm{G}$ or $\mathrm{B}$ texture components. Austenite grain orientations close to 

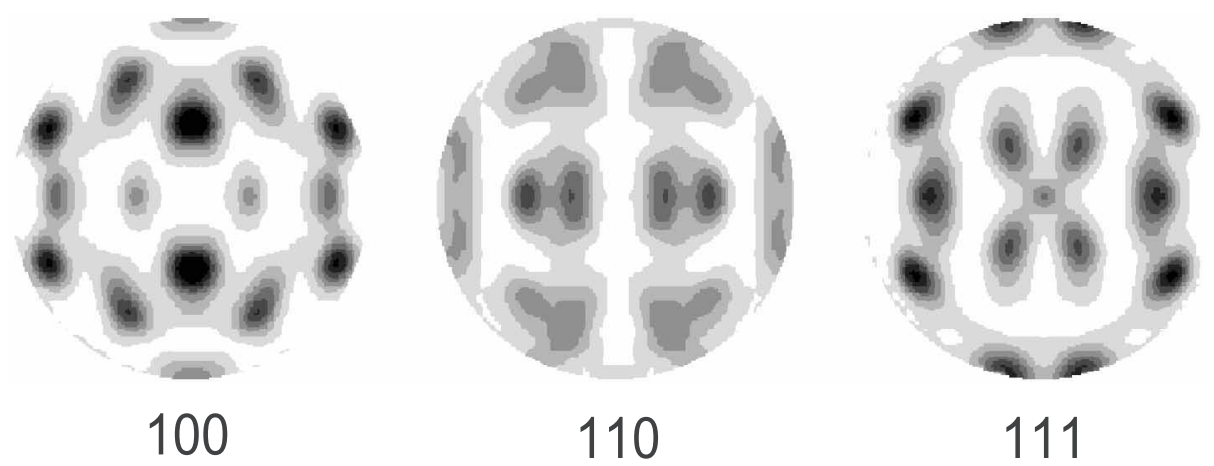

FIGURE 5 Simulated $\alpha$ pole figures based on decomposition of the $\gamma$ starting texture in 3 ideal components, and using the KS orientation relation. Compare to Fig. 2(b).

the dominating $\mathrm{C}$ component, but with $\langle-111\rangle_{\gamma}$ parallel to the $\mathrm{RD}$, have been accounted for by convolution with a one-dimensional Gaussian probability distribution of width $\pm 10^{\circ}$. Comparison with the pole figures in Fig. 2(b) shows reasonable qualitative agreement with the experimental martensite texture. Where significant differences occur they are most likely the result of the idealized starting texture used in the simulation, or they may indicate that not all of the "allowed" variants occur with equal probability. It is also possible that the probability of occurrence of individual observed orientation variants varies between the $\mathrm{C}, \mathrm{G}$ and $\mathrm{B}$ components of the starting texture. Furthermore, the precise orientation relation between the native and transformed phases may deviate slightly from the KS model. The present results are qualitative and should be regarded as a basis for a more quantitative treatment where the probability for a given orientation variant to occur in the texture transformation is obtained from a quantitative fit of a suitably parametrized texture model to the experimentally determined orientation distribution of the transformed $\alpha$ phase. Such a refined procedure will be particularly necessary to decide with greater clarity whether or not the texture transformation of the $\mathrm{C}$ component involves variant selection according to the (lower) sheet sample symmetry rather than the axial symmetry of the applied tensile strain.

\section{CONCLUSIONS}

The strain induced texture transformation from the fcc $\gamma$ phase to the bcc $\alpha$ phase in metastable austenitic stainless steel has been studied on sheet samples exposed to uniaxial tension applied along the rolling direction. Comparison of X-ray pole figures obtained from deformed and undeformed samples shows that all 3 ideal texture components $(\mathrm{C}, \mathrm{G}, \mathrm{B})$ present in the starting $\gamma$ phase transform into martensite. The transformation can be qualitatively described by the Kurdjumov-Sachs orientation relation. Strong variant selection is inferred for the two major $\mathrm{C}$ and $\mathrm{G}$ components. In the case of the dominating $\mathrm{C}$ component the presence of individual orientation variants appears to depend on whether or not the applied strain favors shear in different $\{111\}_{\gamma}$ planes. Variant selection for this component occurs most likely under preservation of the axial symmetry of the applied strain. A Bain-type model assuming that 
orientation variants appear preferentially if they can be related to a compressive component of the applied strain along an fcc cube axis provides a necessary condition for the variant selection observed in the case of the $\mathrm{C}$ component but does not explain why selection occurs also between variants with a common compression axis. A minimum of 3 different ideal orientations present in the $\alpha$ phase are identified as the transformation product of the B component of the starting texture.

\section{Acknowledgments}

This work would not have been possible without the outstanding support of S. W. Robertson, Lawrence Berkeley National Laboratory, L. Lutterotti, University of Trento, Italy, and the BESSRC team at the APS. Use of the Advanced Photon Source was supported by the U.S. Department of Energy, Basic Energy Sciences, Office of Science, under Contract No. W-31-109-Eng-38.

\section{References}

Abe, H. and Ito, K. (1967). Effect of nickel content on the texture of martensite in Fe-Ni alloy rolled above $M_{d}$ temperature. J. Jpn. Inst. Met., 31, 1300-1305.

Angel, T. (1954). Formation of martensite in austenitic stainless steels. J. Iron Steel Inst., 177, 165-174.

Bogers, A.J. and Burgers, W.G. (1964). Partial dislocations on the $\{110\}$ planes in the f.c.c. lattice and the transition of the f.c.c. into the b.c.c. lattice. Acta Metall., 12, 255-261.

Bonarski J., Wrobel M. and Pawlik, K. (1991). Improvement in quantitative phase analysis of textured materials. Scripta Metall. Mater., 25, 1401-1404.

Borik, F. and Richman, R.H. (1967). Preferred transformation in strain-hardened austenite. Trans. Metall. Soc. Soc. AIME, 239, 675-680.

Bronkhorst, C.A., Kalidindi, S.R. and Anand, L. (1992). Polycrystalline plasticity and the evolution of crystallographic texture in fcc metals. Phil. Trans. R. Soc. Lond., 341, 443-477.

Chapellier, Ph., Ray, R.K. and Jonas J.J. (1990). Prediction of transformation textures in steels. Acta Metall. Mater., 38, 1475-1490.

Dahmen, U. (1982). Orientation relationships in precipitation systems. Acta Metall., 30, 63-73.

Ding, P., Inoue T., Imatani, S., Ju, D.Y. and de Vries, E. (2001). Forging process simultion incorporating strain-induced phase transformation by finite volume method. In: Mori, K. (Ed.), Simulation of Materials Processing: Theory, Methods and Applications, pp. 103-108. Swets and Zeitlinger, Lisse, The Netherlands.

Furubayashi, E. (1985). Transformation texture analysis with Bain relation - Monte Carlo simulations. Tetsu-to-Hagané, 71, 1359-1366.

Goodchild, D., Roberts, W.T. and Wilson, D.V. (1970). Plastic deformation and phase transformation in textured austenitic stainless steel. Acta Metall., 18, 1137-1146.

Heidelbach, F., Riekel, C. and Wenk, H.-R. (1999). Quantitative texture analysis of small domains with synchrotron radiation X-rays. J. Appl. Cryst., 32, 841-849.

Higo, Y., Lecroisey, F. and Mori, T. (1974). Relation between applied stress and orientation relationship of a $\alpha$ martensite in stainless steel single crystals. Acta Metall., 22, 313-323.

Jung, V. (1983). Can inherited textures in the b.c.c. phase furnish information about the type of transformation from the f.c.c. phase? J. Appl. Cryst., 16, 535-541.

Kato, M. and Mori, T. (1977). Orientation of martensite formed in $\mathrm{Fe}-23 \mathrm{Ni}-5 \mathrm{Cr}$ crystals under uniaxial stress along [001]. Acta Metall., 25, 951-956.

Lutterotti, L., Matthies, S., Wenk, H.-R., Schultz, A.J. and Richardson, J.W. (1997). Combined texture and structure analysis of deformed limestone from time-of-flight neutron diffraction spectra. J. Appl. Phys., 81, 594-600.

Matthies, S. and Vinel, G.W. (1982). On the reproduction of the orientation distribution function of texturized samples from reduced pole figures using the conception of a conditional ghost correction. Phys. Stat. Solidi (b), 112, K111-114.

Miyaji, H. and Furubayashi, E. (1990). Effect of specimen size on the variant selection in martensitic transformation. Textures and Microstructures, 12, 189-197.

Olsen, G.H. and Jesser, W.A. (1971). The Effect of applied stress on the f.c.c.-b.c.c. Transformation in thin iron films. Acta Metall., 19, 1299-1302. 
Olson, G.B. and Cohen, M. (1975). Kinetics of strain-induced martensitic nucleation. Metall. Trans. A, 6, 791-795.

Patel, J.R. and Cohen, M. (1953). Criterion for the action of applied stress in the martensitic transformation. Acta Metall., 1, 531-538.

Raabe, D. (1997). Texture and microstructure evolution during cold rolling of a strip cast and of a hot rolled austenitic stainless steel. Acta Mater., 45, 1137-1151.

Stone, G. and Thomas, G. (1974). Deformation induced alpha and epsilon martensites in $\mathrm{Fe}-\mathrm{Ni}-\mathrm{Cr}$ single crystals. Metall. Trans., 5, 2095-2102.

Sato, A., Kato, M., Sunaga, Y., Miyazaki, T. and Mori, T. (1980). Stress induced martensitic transformation in Fe-Ni-C alloy single crystals. Acta Metall., 28, 367-376.

Von Dreele, R.B. (1997). Quantitative texture analysis by Rietveld refinement. J. Appl. Cryst., 30, $517-525$.

Wcislak, L., Klein, H., Bunge, H.J., Garbe, U., Tschentscher, T. and Schneider, J.R. (2002). Texture analysis with high-energy synchrotron radiation. J. Appl. Cryst., 35, 82-95. 